\title{
A variational principle for stationary, axisymmetric solutions of Einstein's equations
}

\author{
Sergio Dain \\ Max-Planck-Institut für Gravitationsphysik, Am Mühlenberg 1, 14476 Golm, Germany
}

Received 30 June 2006, in final form 19 September 2006

Published 20 October 2006

Online at stacks.iop.org/CQG/23/6857

\begin{abstract}
Stationary, axisymmetric, vacuum, solutions of Einstein's equations are obtained as critical points of the total mass among all axisymmetric and $(t, \phi)$ symmetric initial data with fixed angular momentum. In this variational principle, the mass is written as a positive definite integral over a spacelike hypersurface. It is also proved that if an absolute minimum exists then it is equal to the absolute minimum of the mass among all maximal, axisymmetric, vacuum, initial data with fixed angular momentum. Arguments are given to support the conjecture that this minimum exists and is the extreme Kerr initial data.

PACS numbers: 04.70.Bw, 04.20.Dw, 04.20.Ex, 04.20.Fy
\end{abstract}

\section{Introduction}

In an axisymmetric, vacuum, gravitational collapse the total angular momentum is an absolutely conserved quantity. Therefore, if we assume, according to the standard picture of the gravitational collapse, that the final state will be a Kerr black hole the following inequality should hold for every axisymmetric, vacuum, asymptotically flat, complete, initial data set:

$$
\sqrt{|J|} \leqslant m
$$

where $m$ is the mass of the data and $J$ is the angular momentum in the asymptotic region. Moreover, the equality in (1) should imply that the data are the slice of the extreme Kerr black hole. A counterexample to (1) will provide a regular vacuum data that do not settle down to a Kerr black hole. For a more detailed discussion of the motivations and relevance of (1) and related inequalities, see $[9,11,15]$.

Inequality (1) is a property of the spacetime and not only of the data, since both quantities $J$ and $m$ are independent of the slicing. It is in fact a property of axisymmetric, vacuum, black holes spacetimes, because a non-zero $J$ (in vacuum) implies a non-trivial topology on the data and this is expected to signal the presence of a black hole. Note, however, that the mass 
in (1) is a global quantity but the angular momentum is a quasilocal quantity because we have assumed axial symmetry. Without axial symmetry we still have $J$ defined as a global quantity at spacelike infinity, but (1) is no longer true in this case. A more subtle question is whether (1) is true, where both $m$ and $J$ are quasilocal quantities, that is, whether (1) is in fact a quasilocal property of the black hole. In general there is no unique definition of quasilocal mass (see the recent review on the subject [20]). However, a remarkable counterexample was found in [1] in which there is a clear quasilocal mass definition (the Komar mass), and inequality (1) is violated at the quasilocal level. It is also important to recall that (1) is false for black holes in higher dimensions (see, for example, [16] and reference therein). Finally, note that there exist analogue inequalities for charge and mass (see [4, 12]) and for spin and mass in anti-de Sitter spacetimes (see [13]).

Inequality (1) suggests the following variational principle.

(i) The extreme Kerr initial data are the absolute minima of the mass among all axisymmetric, vacuum, asymptotically flat and complete initial data with fixed angular momentum.

So far, there is no proof of (1). A promising strategy to prove it is to use the variational formulation (i). In this paper, we will prove the following results, which are a step forward in this direction.

The first result is the following related variational principle.

(ii) The critical points of the mass among all the axisymmetric, $(t, \phi)$ symmetric, asymptotically flat data are the stationary, axisymmetric solutions.

A spacetime is defined to be $(t, \phi)$ symmetric if it is symmetric under a simultaneous change of sign of the time coordinate $t$ and the axial angle $\phi$. Data are called $(t, \phi)$ symmetric if their evolution is a $(t, \phi)$ symmetric spacetime. These data are also known as 'momentarily stationary data' (see [2] for more details). The variational principle (ii) was proved by Bardeen [2], who also included matter in the formulation. It was also studied by Hawking [14] for black holes including boundary terms. However, in all these works the mass is not written as a positive definitive integral (see the discussion of section 8 in [2]). Therefore, it is not possible to relate (ii) with (i) in these formulations. In this paper, we will prove (ii) using the mass formula discovered by Brill [5], which is a positive definitive integral over the slice. Using this formulation of (ii) we will be able to prove the following.

(i') If the absolute minimum of the mass among all axisymmetric, $(t, \phi)$ symmetric, vacuum, asymptotically flat and complete initial data with fixed angular momentum exists, then it is equal to the absolute minimum of the mass among all maximal, axisymmetric (the axial vector is assumed to be surface orthogonal), vacuum, asymptotically flat and complete initial data with fixed angular momentum. Moreover, the absolute minimum is stationary.

That is, we have essentially reduced the variational problem (i) to the $(t, \phi)$ symmetric case. Note that we have included in (i') the conditions that the data are maximal (i.e., the trace of the second fundamental form is zero) and that the axial Killing vector is hypersurface orthogonal on the slice. These are technical assumptions which simplify considerably the analysis, but the statement is expected to be valid without them.

There exist other variational formulations of the stationary, axisymmetric, equations, see $[17,19]$. Particularly interesting in the present context is the variational formulation given by Carter [8] which is based on the Ernst formulation [10]. There exists a remarkable connection between (ii) in the present formulation and Carter's variational principle; we will prove that the Lagrangians differ only by a (singular) boundary term. 


\section{Axially symmetric initial data and Brill proof of the positive mass theorem}

In this section, we review Brill's positive mass theorem for axisymmetric data [5]. The original proof was for time-symmetric data in $\mathbb{R}^{3}$; Bardeen [2] extended it to momentarily stationary data. Here we slightly extend the proof to include maximal data and non-trivial topologies.

An initial data set for Einstein's vacuum equations consists in a 3-manifold $S$, a Riemannian metric $\tilde{h}_{a b}$ and a symmetric tensor field $\tilde{K}^{a b}$ such that the vacuum constraint equations

$$
\begin{aligned}
& \tilde{D}^{b} \tilde{K}_{a b}-\tilde{D}_{a} \tilde{K}=0, \\
& \tilde{R}+\tilde{K}^{2}-\tilde{K}_{a b} \tilde{K}^{a b}=0
\end{aligned}
$$

are satisfied on $S$, where $\tilde{D}_{a}$ and $\tilde{R}$ are the Levi-Civita connection and the Ricci scalar associated with $\tilde{h}_{a b}, \tilde{K}=\tilde{h}^{a b} \tilde{K}_{a b}$, and the indices are moved with the metric $\tilde{h}_{a b}$ and its inverse $\tilde{h}^{a b}$.

We will assume that the initial data are axially symmetric, that is, there exists an axial Killing vector $\eta^{a}$ such that

$$
£_{\eta} \tilde{h}_{a b}=0, \quad £_{\eta} \tilde{K}_{a b}=0,
$$

where $£$ denotes the Lie derivative. The Cauchy development of such initial data will be an axially symmetric spacetime.

The Killing vector $\eta^{a}$ is assumed to be orthogonal with respect to $\tilde{h}_{a b}$ to a family of 2 -surfaces in $S$. Under these conditions, the metric $\tilde{h}_{a b}$ can be characterized by two functions, one is essentially the norm of the Killing vector and the other is a conformal factor on the 2 -surfaces. We make explicit the choice of the free functions as follows. Let $(\rho, z, \phi)$ be local coordinates in $S$ such that the metric has the following form:

$$
\tilde{h}_{a b}=\psi^{4} h_{a b},
$$

where the conformal metric $h_{a b}$ is given by

$$
h=\mathrm{e}^{-2 q}\left(\mathrm{~d} \rho^{2}+\mathrm{d} z^{2}\right)+\rho^{2} \mathrm{~d} \varphi^{2},
$$

and $q, \psi$ are functions which depend only on $z$ and $\rho$ with $\psi>0$. The vector $\eta^{a}=(\partial / \partial \varphi)^{a}$ is a Killing vector of both metrics $\tilde{h}_{a b}$ and $h_{a b}$. The norm of $\eta^{a}$ with respect to the physical metric will be denoted by $X$, (i.e. $X=\eta^{a} \eta^{b} \tilde{h}_{a b}=\psi^{4} \rho^{2}$ ), and the norm of $\eta^{a}$ with respect to the conformal metric is given by $\rho^{2}=\eta^{a} \eta^{b} h_{a b}$.

We define the following quantity:

$$
J(\Sigma)=\oint_{\Sigma} \tilde{\pi}_{a b} \eta^{a} \tilde{n}^{b} \mathrm{~d} s_{\tilde{h}},
$$

where $\tilde{\pi}_{a b}=\tilde{K}_{a b}-\tilde{h}_{a b} \tilde{K}, \Sigma$ is any closed 2-surface, $\tilde{n}^{a}$ is the unit normal vector to $\Sigma$ with respect to $\tilde{h}_{a b}$ and d $s_{\tilde{h}}$ is the area element of $\Sigma$ with respect to $\tilde{h}_{a b}$. Equation (2) and the Killing equation imply that the vector $\tilde{\pi}_{a b} \eta^{a}$ is divergence free. If $\Sigma$ is the boundary of some compact domain $\Omega \subset S$, by the Gauss theorem, we have $J(\Sigma)=0$. For example, if $S=\mathbb{R}^{3}$ then $J(\Sigma)=0$ for all $\Sigma$. In an asymptotically flat data, $J\left(\Sigma_{\infty}\right)$ gives the total angular momentum, where $\Sigma_{\infty}$ is any closed surface in the asymptotic region. Then, the angular momentum will be zero unless $\Sigma_{\infty}$ is not the boundary of some compact domain contained in $S$.

In order to have non-zero angular momentum, we will allow $S$ to have many asymptotic ends ${ }^{1}$. Let $i_{k}$ be a finite number of points in $\mathbb{R}^{3}$. The manifold $S$ is assumed to be $\mathbb{R}^{3} \backslash \sum_{k} i_{k}$. The points $i_{k}$ will represent the extra asymptotic ends; at those points we will impose singular

\footnotetext{
1 There is an interesting alternative (not included here) discussed in [11] to allow non-zero angular momentum: the interior of the manifold is assumed to be compact and non-simply connected with a pseudo axial Killing vector. A pseudo axial Killing vector is a rotational Killing vector defined only up to sign, see [11] for the precise definition.
} 
boundary conditions for $\psi$. To be consistent with the axial symmetry assumption, the points $i_{k}$ should be located on the axis $\rho=0$.

In addition to axial symmetry we will assume that the data are maximal:

$$
\tilde{K}=0 \text {. }
$$

By equation (3), this implies that $\tilde{R}$ is non-negative; this will be essential in order to extend Brill's proof to non-time symmetric data.

Define the conformal second fundamental form by $K^{a b}=\psi^{10} \tilde{K}^{a b}$. Using (8) and (4) we obtain

$$
£_{\eta} K_{a b}=0, \quad K=0 .
$$

The constraint equations (2)-(3) can be written as equations for $K_{a b}$ and $\psi$ using the well-known conformal method (see, for example, [3] and reference therein)

$$
\begin{aligned}
& D_{a} K^{a b}=0, \\
& D^{a} D_{a} \psi-\frac{1}{8} R \psi=-\frac{1}{8} K_{a b} K^{a b} \psi^{-7},
\end{aligned}
$$

where $D_{a}$ and $R$ are the Levi-Civita connection and the Ricci scalar associated with the conformal metric $h_{a b}$. In these equations, the indices are moved with the conformal metric $h_{a b}$ and its inverse $h^{a b}$.

The function $q$ is assumed to be smooth with respect to the coordinates $(\rho, z)$. At the axis, we impose the regularity condition

$$
q(\rho=0, z)=0 .
$$

Note that condition (12) includes the points $i_{k}$. These points are assumed to be regular points of the conformal metric $h_{a b}$, that is, $h_{a b}$ is well defined in $\mathbb{R}^{3}$.

We assume the following fall-off condition at infinity:

$$
q=o\left(r^{-1}\right), \quad q_{, r}=o\left(r^{-2}\right),
$$

where $r=\sqrt{\rho^{2}+z^{2}}$ and a comma denotes partial derivatives. This fall-off conditions imply that the total mass of the conformal metric $h_{a b}$ is zero.

At infinity, the conformal factor $\psi$ and the conformal second fundamental form satisfy

$$
\psi-1=O\left(r^{-1}\right), \quad \psi_{, r}=O\left(r^{-2}\right)
$$

and

$$
K_{a b}=O\left(r^{-2}\right) .
$$

Under these assumptions the total mass of the physical metric is given by

$$
m=\frac{-1}{2 \pi} \lim _{r \rightarrow \infty} \oint_{\Sigma_{r}} n^{a} D_{a} \psi \mathrm{d} s_{h},
$$

where $\Sigma_{r}$ are the 2-spheres $r=$ constant, $n^{a}$ is the unit normal, with respect to $h_{a b}$, pointed outwards and $\mathrm{d} s_{h}$ is the area element of $\Sigma$ with respect to $h_{a b}$.

The Ricci scalar $R$ of the conformal metric (6) is given by

$$
R=2 \mathrm{e}^{2 q}\left(q_{, \rho \rho}+q_{, z z}\right) .
$$

We have the important equation

$$
\int_{\mathbb{R}^{3}} R \mathrm{~d} \mu_{h}=0
$$


where $\mathrm{d} \mu_{h}$ is the volume element of the metric $h_{a b}$ To prove this, note that $\mathrm{d} \mu_{h}=$ $\rho \mathrm{e}^{-2 q} \mathrm{~d} \rho \mathrm{d} z \mathrm{~d} \phi$, then

$$
\begin{aligned}
\int_{\mathbb{R}^{3}} R \mathrm{~d} \mu_{h} & =4 \pi \int_{0}^{\infty} \mathrm{d} \rho \int_{-\infty}^{\infty} \mathrm{d} z\left(q_{, \rho \rho}+q_{, z z}\right) \rho \\
& =4 \pi \int_{0}^{\infty} \mathrm{d} \rho \int_{-\infty}^{\infty} \mathrm{d} z\left(\left(\rho q_{, \rho}-q\right)_{, \rho}+\left(\rho q_{, z}\right)_{, z}\right),
\end{aligned}
$$

we use the divergence theorem in two dimension to transform this volume integral in a boundary integral over the axis $\rho=0$ and infinity. The boundary integral at the axis vanishes since $q$ satisfies (12), and at infinity it also vanishes because of (13).

Since $\lim _{r \rightarrow \infty} \psi=1$, we have an equivalent expression for the mass

$$
m=\frac{-1}{2 \pi} \lim _{r \rightarrow \infty} \oint_{\Sigma_{r}} \frac{n^{a} D_{a} \psi}{\psi} \mathrm{d} s_{h} .
$$

We use the identity

$$
D_{a}\left(\frac{D^{a} \psi}{\psi}\right)=\frac{D^{a} D_{a} \psi}{\psi}-\frac{D_{a} \psi D^{a} \psi}{\psi^{2}}
$$

the constraint equations (11), (18) and the mass formula (21) to obtain the final expression

$$
m=\frac{1}{2 \pi} \int_{\mathbb{R}^{3}}\left(\frac{K^{a b} K_{a b}}{8 \psi^{8}}+\frac{D_{a} \psi D^{a} \psi}{\psi^{2}}\right) \mathrm{d} \mu_{h},
$$

which is definite positive. To obtain (23) from (22), we have assumed that the boundary integral around the singular points $i_{k}$ vanishes, that is

$$
\lim _{r_{k} \rightarrow 0} \oint_{\Sigma_{r_{k}}} \frac{n^{a} D_{a} \psi}{\psi} \mathrm{d} s_{h}=0
$$

where $r_{k}$ is the distance to the point $i_{k}$. This condition (which is, of course, trivially satisfied when the topology of the physical data is $\mathbb{R}^{3}$ ) allows for a singular behaviour of $\psi$ at $i_{k}$, which in particular includes the case where $i_{k}$ are asymptotically flat ends. Near an asymptotically flat end $i_{k}$, the conformal factor satisfies $\psi=O\left(r_{k}^{-1}\right), \psi_{, r}=O\left(r_{k}^{-2}\right)$ which imply (24). To illustrate this, consider the following two examples.

The Schwarzschild initial data in isotropic coordinates are time-symmetric $\left(K_{a b}=0\right)$ and conformally flat $(q=0)$. In this case, we have one point $i_{0}$ located at the origin and the conformal factor is given by

$$
\psi=1+\frac{m_{0}}{2 r},
$$

where $m_{0}$ is the Schwarzschild mass. We have

$$
\begin{aligned}
m & =2 \int_{0}^{\infty} \frac{\left(\psi_{, r}\right)^{2}}{\psi^{2}} r^{2} \mathrm{~d} r \\
& =m_{0} .
\end{aligned}
$$

Note that the integral is taken over the space spanned by the two asymptotic regions.

The second example is the axisymetric Brill-Lindquist [6] initial data. In this case, the data are also time-symmetric and conformally flat, but here we have $n$ ends $i_{k}$ and the conformal factor is given by

$$
\psi=1+\sum_{k}^{n} \frac{m_{k}}{2 r_{k}}
$$


where $m_{k}$ are arbitrary positive constants. The conformal factor (28) satisfies (24) and we have that

$$
m=\frac{1}{2 \pi} \int_{\mathbb{R}^{3}}\left(\frac{D_{a} \psi D^{a} \psi}{\psi^{2}}\right) \mathrm{d} \mu_{h}=\sum_{k}^{n} m_{k} .
$$

In the non-time-symmetric case, we have assumed that the integral of $K^{a b} K_{a b} \psi^{-8}$ over $\mathbb{R}^{3}$ is bounded. At infinity, the integral converges because assumptions (15) and (14). At the points, $i_{k}$ the conformal second fundamental form will, in general, be singular. However, the integral will be bounded because the singular behaviour of $K_{a b}$ will be cancelled out by the singular behaviour of $\psi$. For example, in the asymptotically flat case, $K_{a b}=O\left(r_{k}^{-4}\right)$ near $i_{k}$ and then we have that $K^{a b} K_{a b} \psi^{-8}$ is bounded. In appendix A, we prove that Kerr initial data satisfy these conditions.

\section{The variational principle}

In integral (23), the mass depends on the metric variables $\psi, q$ (the function $q$ appears in the volume element and in the indices contractions) and on the conformal second fundamental form $K^{a b}$. These functions are not independent; they have to satisfy the constraint equations (10) and (11). In order to formulate the variational principle, we want to express the mass in terms of functions that can be freely varied. We analyse first the conformal second fundamental form $K^{a b}$ and the constraint (10).

Consider the following vector field $S^{a}$ :

$$
S_{a}=K_{a b} \eta^{b}-\rho^{-2} \eta_{a} K_{b c} \eta^{b} \eta^{c} .
$$

Using equations (9), (10) and the Killing equation for $\eta^{a}$, it follows that $S^{a}$ satisfies

$$
£_{\eta} S^{a}=0, \quad S^{a} \eta_{a}=0, \quad D_{a} S^{a}=0 .
$$

From (7), we deduce an equivalent expression for the total angular momentum

$$
J=-\frac{1}{8 \pi} \oint_{\Sigma_{\infty}} S_{a} n^{a} \mathrm{~d} s_{h}
$$

where we have used that the second term on the right-hand side of (30) does not contribute to the angular momentum because we can always chose a closed surface at infinity such that $n^{a} \eta_{a}=0$.

The conformal metric $h_{a b}$ can be decomposed into

$$
h_{a b}=q_{a b}+\rho^{-2} \eta_{a} \eta_{b},
$$

where

$$
q_{a b} \equiv \mathrm{e}^{-2 q}\left(\mathrm{~d} \rho^{2}+\mathrm{d} z^{2}\right)
$$

is the intrinsic metric of the planes orthogonal to $\eta^{a}$. Using this decomposition and the definition of $S^{a}$, we obtain the following expression for the square of the conformal second fundamental form:

$$
K^{a b} K_{a b}=K_{a b} K_{c d} q^{a c} q^{b d}+\rho^{-4}\left(K_{a b} \eta^{a} \eta^{b}\right)^{2}+2 \rho^{-2} S^{a} S_{a}
$$

The two first terms on the right-hand side of this equation are positive, then we have

$$
K^{a b} K_{a b} \geqslant 2 \rho^{-2} S^{a} S_{a} .
$$

Equations (32) and (36) are important because they show that $S^{a}$ contains the angular momentum of $K^{a b}$ and its square is a lower bound for the square of $K^{a b}$. 
We define the tensor

$$
\bar{K}^{a b}=\frac{2}{\eta} S^{(a} \eta^{b)}
$$

we have

$$
\bar{K}^{a b} \bar{K}_{a b}=\frac{2 S^{a} S_{a}}{\rho^{2}} .
$$

It is interesting to note (but we will not make use of it) that this tensor is trace free and divergence free. To prove this, we use the Killing equation $D_{(a} \eta_{b)}=0$, the fact that $\eta^{a}$ is hypersurface orthogonal (i.e., it satisfies $D_{a} \eta_{b}=-\eta_{[a} D_{b]} \ln \eta$ ) and equations (31).

Data will be $(t, \phi)$ symmetric if and only if the following conditions hold (see [2]):

$$
K_{a b} q^{a c} q^{b d}=0, \quad K_{a b} \eta^{a} \eta^{b}=0 .
$$

This is equivalent to $K^{a b}=\bar{K}^{a b}$.

The vector $S^{a}$ can be expressed in terms of a free potential. Define the rescaled vector $s^{a}$ by

$$
s^{a}=\mathrm{e}^{-2 q} S^{a}
$$

then

$$
£_{\eta} s^{a}=0, \quad s^{a} \eta_{a}=0, \quad \partial_{a} s^{a}=0,
$$

where $\partial_{a}$ is the connexion with respect to the flat metric

$$
\delta=\mathrm{d} \rho^{2}+\mathrm{d} z^{2}+\rho^{2} \mathrm{~d} \varphi^{2},
$$

and in equation (41) the indices are moved with this metric and its inverse. The same will apply to all the equations from now on: all of them will be given in terms of the flat metric $\delta_{a b}$ and its connexion $\partial_{a}$.

An arbitrary vector $s^{a}$, which satisfies equations (41), can be written in terms of a potential $Y$ in the following form:

$$
s^{a}=\frac{1}{2 \rho^{2}} \epsilon^{a b c} \eta_{b} \partial_{c} Y,
$$

where $\epsilon_{a b c}$ is the volume element of the flat metric (42) and $£_{\eta} Y=0$. The motivation of the normalization factor $1 / 2$ in (43) will be clear in the following section. We have the relation

$$
\bar{K}^{a b} \bar{K}_{a b}=\frac{2 s^{a} s_{a}}{\rho^{2}}=\frac{\partial^{a} Y \partial_{a} Y}{2 \rho^{4}} .
$$

Angular momentum (31) is given in terms of the potential $Y$ by

$$
J=\frac{1}{8}(Y(\rho=0,-z)-Y(\rho=0, z)),
$$

where $z$ is taken to be larger than the location of any point $i_{k}$.

Motivated by Brill's formula (23), we define the mass functional as follows:

$$
\mathcal{M}(v, Y)=\frac{1}{32 \pi} \int_{\mathbb{R}^{3}}\left(16 \partial_{a} v \partial^{a} v+\rho^{-4} \mathrm{e}^{-8 v} \partial^{a} Y \partial_{a} Y\right) \mathrm{d} \mu_{0},
$$

where $v=\ln \psi$ and $\mathrm{d} \mu_{0}$ is the flat volume element. Note that in integral (46) the metric function $q$ does not appear.

From equations (23) and (44), we see that for every axisymmetric and $(t, \phi)$ symmetric data we have $m=\mathcal{M}(v, Y)$. From (36), we see that for every axisymmetric, maximal data, we have

$$
m \geqslant \mathcal{M}(v, Y)
$$


We emphasize that the functions $(v, Y)$ can be computed for an arbitrary axisymmetric data (in the construction of the potential $Y$ we have not used the maximal condition) and then the functional $\mathcal{M}(v, Y)$ can be also calculated for arbitrary data (provided, of course, the integral is well defined). However, only for maximal data, we can use the Brill formula (23) to conclude (47), and only for $(t, \phi)$ symmetric data we have that $\mathcal{M}(v, Y)$ is in fact the mass.

For the present calculations it is more convenient to write the functional $\mathcal{M}$ in the form (46), where the axial symmetry is not explicit. For completeness, we also write it in a manifest axisymmetric form

$$
\mathcal{M}(v, Y)=\frac{1}{16} \int_{0}^{\infty} \mathrm{d} \rho \int_{-\infty}^{\infty} \mathrm{d} z\left(16 \rho\left(v_{, z}^{2}+v_{, \rho}^{2}\right)+\rho^{-3} \mathrm{e}^{-8 v}\left(Y_{, z}^{2}+Y_{, \rho}^{2}\right)\right) .
$$

Let us define $\mathcal{A}$ as the set of all functions $(v, Y)$ such that integral (46) is bounded. Although $\mathcal{M}(v, Y)$ is well defined in $\mathcal{A}$, not for every function in $\mathcal{A}$ we will have that $\mathcal{M}(v, Y)$ is equal to the mass of some $(t, \phi)$ symmetric initial data. This is a subtle and important point; let us discuss it in detail. We have seen that all axisymmetric and $(t, \phi)$ symmetric data can be generated by three functions $(v, q, Y)$. They are coupled by the Hamiltonian constraint (3). In coordinates, this equation is given by

$$
4 \frac{\Delta \psi}{\psi}-\left(q_{, \rho \rho}+q_{, z z}\right)=-\frac{\partial^{a} Y \partial_{a} Y}{4 \rho^{4} \psi^{8}},
$$

where $\Delta$ is the flat Laplacian with respect to (42). For given $(v, Y)($ remember that $v=\ln \psi)$ this is a linear, two-dimensional Poisson equation for $q$. The delicate points are the boundary conditions. In order to obtain Brill's formula, we have required that $q$ satisfies (12) and (13). But we cannot impose these two equations as boundary conditions for a two-dimensional Poisson equation. Let us say that we impose (12) and we ask for solutions which fall off at infinity. This problem can be solved with an explicit Green function. However, in general, the fall-off of the solution will be $q=O\left(r^{-1}\right)$ which is weaker than (13). Only for some particular source functions $(v, Y)$, the solution $q$ will satisfy (13). Let us denote by $\mathcal{A}_{1}$ the subset of $\mathcal{A}$ of those functions $(v, Y)$ such that the solution $q$ of equation (49) satisfies (12) and (13). Only for functions in $\mathcal{A}_{1}$ the functional $\mathcal{M}(v, Y)$ can be written as the boundary integral (16) and hence gives the mass of some initial data. A function $v$ of compact support (such that $\psi=1$ near infinity) is an example of a function which is in $\mathcal{A}$ but not in $\mathcal{A}_{1}$ (we can take $Y=0)$, since in this case clearly $\mathcal{M}(v, Y)$ is strictly positive and the boundary integral (16) is zero.

We want to make variations of $\mathcal{M}(v, Y)$. At first sight, it appears that the appropriate set for admissible functions is $\mathcal{A}_{1}$ and not $\mathcal{A}$. However, it seems to be difficult to characterize $\mathcal{A}_{1}$. It is known how to characterize the set of those $q$ such that (49) has a solution $\psi$ (for an, essentially, arbitrary $Y$ ) which satisfies (14); in this case a nonlinear equation must be solved (see [7] and [18]). However, this set is not very useful in the present context since for the Brill formula it is natural to use $(v, Y)$ as independent functions and not $(q, Y)$. Instead, what we will do is to take $\mathcal{A}$ as the set of admissible functions. Remarkably, it will turn out that the critical equations in this bigger set are only the stationary, axially symmetric equations.

Let $\alpha$ and $y$ be compact supported functions in $\mathbb{R}^{3}$ with support in $S$ and such that the support of $y$ does not contain the axis. By equation (45) we see that this condition implies that the perturbation $Y+y$ does not change the angular momentum of $Y$. Define

$$
\mathrm{i}(\epsilon)=\mathcal{M}(v+\epsilon \alpha, Y+\epsilon y) \text {. }
$$

The first variation of $\mathcal{M}(v, Y)$ is given by

$\mathrm{i}^{\prime}(0)=\frac{1}{16 \pi} \int_{\mathbb{R}^{3}}\left(16 \partial_{a} v \partial^{a} \alpha-4 \alpha \rho^{-4} \mathrm{e}^{-8 v} \partial^{a} Y \partial_{a} Y+\rho^{-4} \mathrm{e}^{-8 v} \partial^{a} Y \partial_{a} y\right) \mathrm{d} \mu_{0}$, 
where a prime denotes derivative with respect to $\epsilon$. Integrating by parts, we obtain that the condition

$$
i^{\prime}(0)=0
$$

for all $\alpha$ and $y$, is equivalent to the following Euler-Lagrange equations:

$$
\begin{aligned}
& 4 \Delta v+\rho^{-4} \mathrm{e}^{-8 v} \partial^{a} Y \partial_{a} Y=0, \\
& \partial^{a}\left(\rho^{-4} \mathrm{e}^{-8 v} \partial_{a} Y\right)=0 .
\end{aligned}
$$

The second variation is given by

$\mathrm{i}^{\prime \prime}(0)=\frac{1}{16 \pi} \int_{\mathbb{R}^{3}}\left\{16 \partial_{a} \alpha \partial^{a} \alpha+\left(32 \alpha^{2} \partial^{a} Y \partial_{a} Y-16 \alpha \partial^{a} Y \partial_{a} y+\partial^{a} y \partial_{a} y\right) \rho^{-4} \mathrm{e}^{-8 v}\right\} \mathrm{d} \mu_{0}$.

There is an equivalent way of deducing equations (53)-(54). Instead of taking $Y$ as variable we take the vector $s^{a}$, which should satisfy the constraints (41). The mass functional is given by

$$
\mathcal{M}(v, s)=\frac{1}{8 \pi} \int_{\mathbb{R}^{3}}\left(4 \partial_{a} v \partial^{a} v+\rho^{-2} \mathrm{e}^{-8 v} s^{a} s_{a}\right) \mathrm{d} \mu_{0} .
$$

Let $\gamma^{a}$ be a compact supported vector in $S$ such that the support of $\gamma^{a}$ does not contain the axis. We assume that $\gamma^{a}$ satisfies the constraint

$$
\partial_{a} \gamma^{a}=0 \text {. }
$$

We define $\mathrm{i}$ in analogous way as in (50). The first variation is given by

$$
\mathrm{i}^{\prime}(0)=\frac{1}{4 \pi} \int_{\mathbb{R}^{3}}\left(4 \partial_{a} v \partial^{a} \alpha-4 \alpha \rho^{-2} \mathrm{e}^{-8 v} s^{a} s_{a}+\rho^{-2} \mathrm{e}^{-8 v} s^{a} \gamma_{a}\right) \mathrm{d} \mu_{0},
$$

integrating by parts we get

$$
\mathrm{i}^{\prime}(0)=\frac{1}{4 \pi} \int_{\mathbb{R}^{3}}\left(-4 \alpha\left(\Delta v+\rho^{-2} \mathrm{e}^{-8 v} s^{a} s_{a}\right)+\rho^{-2} \mathrm{e}^{-8 v} s^{a} \gamma_{a}\right) \mathrm{d} \mu_{0} .
$$

From this, we deduce the Euler-Lagrange equations

$$
\begin{aligned}
& \Delta v+\rho^{-2} \mathrm{e}^{-8 v} s^{a} s_{a}=0, \\
& \rho^{-2} \mathrm{e}^{-8 v} s_{a}=\frac{1}{2} \partial_{a} \Omega,
\end{aligned}
$$

for some function $\Omega$. Equation (60) follows because we can make arbitrary variations in $\alpha$. On the other hand, variations in $\gamma^{a}$ should satisfy the constraint (57). Writing $\gamma^{a}$ as the curl of an arbitrary vector and integrating by parts we get

$$
\partial_{[a} H_{b]}=0,
$$

where

$$
H_{a}=\rho^{-2} s_{a} \mathrm{e}^{-8 v}
$$

Equation (62) is equivalent to (61). Using the constraint $\partial_{a} s^{a}=0$, we deduce the following equations which do not involve $s^{a}$

$$
\begin{aligned}
& 4 \Delta v+\rho^{2} \mathrm{e}^{8 v} \partial^{a} \Omega \partial_{a} \Omega=0, \\
& \partial^{a}\left(\rho^{2} \mathrm{e}^{8 v} \partial_{a} \Omega\right)=0 .
\end{aligned}
$$


Equations (64)-(65) are equivalent to equations (53)-(54), the relation between $\Omega$ and $Y$ is given by

$$
\partial_{a} \Omega=\rho^{-4} \mathrm{e}^{-8 v} \epsilon_{a b c} \eta^{b} \partial^{c} Y .
$$

In the following section, we will prove that these equations are precisely the stationary, axisymmetric, vacuum equations. This will provide also an interpretation for the potential $Y$ and the velocity $\Omega$ in the stationary case. Note that $Y$ is defined for arbitrary data; in contrast $\Omega$ is only defined for solutions of the critical equations, that is, for stationary axisymmetric data.

If we take $Y=0$, then these equations reduce to

$$
\Delta v=0,
$$

which is Weyl equation for axisymmetric, static, spacetimes. This is of course consistent with the results that we are going to prove in the following section. However, it is important to note that the Schwarzschild data in the form (25) do not satisfy (67). Schwarzschild satisfies (67) in Weyl coordinates where $\bar{v}$ and the metric function $\bar{q}$ are given by

$$
\bar{v}=-\frac{1}{4} \ln \left(\frac{\bar{r}_{+}+\bar{r}_{-}-2 m}{\bar{r}_{+}+\bar{r}_{-}+2 m}\right), \quad \bar{q}=\frac{1}{2} \ln \left(\frac{\left(\bar{r}_{+}+\bar{r}_{-}\right)^{2}-4 m^{2}}{4 \bar{r}_{+} \bar{r}_{-}}\right),
$$

with $\bar{r}_{ \pm}^{2}=\bar{\rho}^{2}+(\bar{z} \pm m)^{2}$. The relation with the isotropic coordinates $(r, \theta)$ used in (25) is

$$
\bar{\rho}=\rho\left(1-\frac{m^{2}}{4 r^{2}}\right), \quad \bar{z}=z\left(1+\frac{m^{2}}{4 r^{2}}\right),
$$

where $z=r \cos \theta$ and $\rho=r \sin \theta$. Since $X$ is a scalar independent of coordinates we have $X=\rho^{2} \psi^{4}=\bar{\rho}^{2} \bar{\psi}^{4}$. The function $\bar{q}$ satisfies our assumptions (12) and (13); however the conformal factor $\bar{\psi}=\mathrm{e}^{\bar{v}}$ does not satisfies (24). The conformal factor is singular on the rod $\bar{\rho}=0,-m \leqslant \bar{z} \leqslant m$ (which represents the horizon of Schwarzschild data) and not just on singular points $i_{k}$. The integral $\mathcal{M}(\bar{v}, 0)$ diverges. Note that $\mathbb{R}^{3}$ in Weyl coordinates $(\bar{\rho}, \bar{z})$ represent the exterior of the black holes, in contrast to coordinates $(\rho, z)$ where $\mathbb{R}^{3}$ represent both asymptotic regions.

\section{Stationary axisymmetric fields}

The spacetime metric of a vacuum, stationary and axially symmetric spacetime can be written, in Weyl coordinates, in the following form (see, for example, [21])

$$
g=-V(\mathrm{~d} t-\sigma \mathrm{d} \phi)^{2}+V^{-1}\left[\rho^{2} \mathrm{~d} \phi^{2}+\mathrm{e}^{2 \gamma}\left(\mathrm{d} \rho^{2}+\mathrm{d} z^{2}\right)\right],
$$

where the functions $V, \sigma$ and $\gamma$ depend only on $(\rho, z)$. The two Killing vectors are

$$
\xi^{\mu}=\left(\frac{\partial}{\partial t}\right)^{\mu}, \quad \eta^{\mu}=\left(\frac{\partial}{\partial \phi}\right)^{\mu},
$$

they define the scalars

$$
V=-\xi^{\mu} \xi^{v} g_{\mu \nu}, \quad X=\eta^{\mu} \eta^{\nu} g_{\mu \nu}, \quad W=\eta^{\nu} \xi^{\mu} g_{\nu \mu},
$$

where $\mu, v$ are spacetime indices. We have the following relations:

$$
W=V \sigma, \quad \rho^{2}=V X+W^{2} .
$$

The vacuum field equations are given by

$$
\partial^{a}\left(V^{-1} \partial_{a} V+\rho^{-2} V^{2} \sigma \partial_{a} \sigma\right)=0,
$$




$$
\partial^{a}\left(\rho^{-2} V^{2} \partial_{a} \sigma\right)=0 .
$$

We want to prove that these equations are equivalent to equations (64)-(65). We first compute the relation between $(V, \sigma)$ and $(v, \Omega)$.

Take an slice $t=$ constant of the metric (70). The intrinsic metric of this surface is given by

$$
\tilde{h}=V^{-1}\left(\rho^{2}-\sigma^{2} V^{2}\right) \mathrm{d} \phi^{2}+V^{-1} \mathrm{e}^{2 \gamma}\left(\mathrm{d} \rho^{2}+\mathrm{d} z^{2}\right) .
$$

To write this metric in the form (5)-(6) set

$$
\psi^{4}=\frac{\left(\rho^{2}-V^{2} \sigma^{2}\right)}{V \rho^{2}}=\frac{X}{\rho^{2}}=\frac{X}{\left(V X+W^{2}\right)},
$$

and

$$
\mathrm{e}^{2 q}=\frac{\mathrm{e}^{2 \gamma} \rho^{2}}{\left(\rho^{2}-V^{2} \sigma^{2}\right)}=\frac{\mathrm{e}^{2 \gamma} \rho^{2}}{V X} .
$$

From (76), we deduce

$$
v(V, \sigma)=\frac{1}{4} \ln \frac{\left(\rho^{2}-V^{2} \sigma^{2}\right)}{V \rho^{2}} .
$$

In order to compute $\Omega(v, \sigma)$, we need to calculate the second fundamental form of this foliation. The lapse and the shift of the foliation $t=$ constant are given by

$$
N=\frac{\rho}{\sqrt{X}}=\psi^{-2}, \quad N_{a}=W(\mathrm{~d} \phi)_{a},
$$

and the second fundamental form is

$$
\tilde{K}_{a b}=-\frac{1}{2 N} \tilde{D}_{(a} N_{b)}
$$

We write $N_{a}$ in terms of the Killing vector $\eta^{a}$, as in the previous section we define $\tilde{\eta}_{a}=\tilde{h}_{a b} \eta^{b}$, where $\tilde{h}_{a b}$ is given by (75), then we have

$$
\tilde{\eta}_{a}=\frac{\left(\rho^{2}-V^{2} \sigma^{2}\right)}{V}(\mathrm{~d} \phi)_{a} .
$$

Using this expression we write $N_{a}$ as

$$
N_{a}=\Omega \tilde{\eta}_{a}
$$

where $\Omega$ is given by

$$
\Omega(V, \sigma)=\frac{V^{2} \sigma}{2\left(\rho^{2}-V^{2} \sigma^{2}\right)}=\frac{W}{X} .
$$

The scalar $\Omega$ can be interpreted as the angular velocity of the locally non-rotating observers (see [2] and also [21] p 187). We want to prove that this function $\Omega$ is precisely the potential $\Omega$ of the previous section. In order to see this, let us compute the vector $s^{a}$

$$
s_{a}=\eta^{b} K_{a b}=\psi^{2} \eta^{b} \tilde{K}_{a b}=\frac{1}{2 N} \psi^{2} X \partial_{a} \Omega=\frac{1}{2} \psi^{8} \rho^{2} \partial_{a} \Omega .
$$

Where we have used $s_{a}=s^{b} \delta_{a b}=S^{b} h_{a b}$. Equation (84) is identical to equation (61).

Using relations (78) and (83), after a long but straightforward computation, we conclude that equations (73) and (74) for the functions $(V, \sigma)$ are equivalent to equations $(60)-(61)$ for $(v, \Omega)$. 
There is another way to prove the equivalence with the stationary equations, using the potential $Y$. We replace $v$ by $X$, that is we consider $X, Y$ as variables. From equation (76) we get

$$
v=\frac{1}{4} \ln X-\frac{1}{2} \ln \rho .
$$

Take the functional $\mathcal{M}$ defined in (46), but let us perform the integral on a bounded domain $B$, in terms of the variables $X, Y$ we get

$\mathcal{M}(X, Y)=\mathcal{M}^{\prime}(X, Y)-\frac{1}{8 \pi} \int_{B} \ln \left(\frac{\rho}{X}\right) \Delta \ln \rho \mathrm{d} \mu_{0}+\oint_{\partial B} \rho^{-1} \ln \left(\frac{\rho}{X}\right) n^{a} \partial_{a} \rho \mathrm{d} s$,

where we have defined

$$
\mathcal{M}^{\prime}(X, Y)=\frac{1}{32 \pi} \int_{B}\left(\frac{\partial_{a} X \partial^{a} X+\partial^{a} Y \partial_{a} Y}{X^{2}}\right) \mathrm{d} \mu_{0} .
$$

But we have

$$
\Delta \ln \rho=0
$$

for $\rho \neq 0$. Then $\mathcal{M}$ and $\mathcal{M}^{\prime}$ differ only by a boundary term. Hence, they give the same EulerLagrange equations. Note, however, that the boundary term is singular at the axis $\rho=0$ : if we take a cylinder $\rho=$ constant near the axis, we have $X=O\left(\rho^{2}\right), \mathrm{d} s=\rho \mathrm{d} z \mathrm{~d} \phi, n^{a} \partial_{a} \rho=1$, then the boundary term diverges like $O(\ln \rho)$ as $\rho \rightarrow 0$.

In [8], Carter formulates a variational principle for the axisymmetric, stationary equations. This formulation is, essentially, a modification of the [10] formulation in which the norm of the axial Killing vector (and not of the stationary one) is taken to be the principal variable.

Carter's Lagrangian is precisely $\mathcal{M}^{\prime}$ (we use the same notation for $X$ and $Y$, this is the reason for the normalization factor $1 / 2$ in (43)). In [8], it is proved that the critical equations of $\mathcal{M}^{\prime}$ are the stationary, axisymmetric equations. Therefore, the same is valid for $\mathcal{M}$. There are, however, some important points that we want to stress.

If we ignore boundary terms, then equation (86) provides an interpretation for Carter Lagrangian. Also, it gives an interpretation of the space of admissible functions in which the variations are made for the following reason. In Carter's formulation $Y$ is defined in terms of $W$ and $X$ by

$$
\epsilon_{a b c} \eta^{b} \partial^{c} Y=X \partial_{a} W-W \partial_{a} X
$$

This equation can easily be obtained from (66), (76) and (83). That is, $Y$ is defined only for stationary axisymmetric spacetimes. From the discussion of section 3, we have seen that $Y$ can be defined for arbitrary, axisymmetric data, and the variations of $Y$ and $X$ are in fact variation among axisymmetric and $(t, \phi)$ symmetric data.

Let us consider boundary terms. The behaviour of $X$ near the axis implies that $\mathcal{M}^{\prime}$ is singular if the domain of integration includes the axis. On the other hand we have seen that $\mathcal{M}$ is finite. In particular, in appendix A we have explicitly checked that Kerr initial data in quasi-isotropic coordinates satisfy all our assumptions and then $\mathcal{M}$ is finite and equal to the mass for Kerr. However, it is important to note that the relevant domains of integration are different in Carter's formulation and in the present one. In [8], the domain is the black hole exterior region, in which the inner boundary is the horizon. In section 3 , we have not included any inner boundary conditions; the domain of integration is the whole manifold which can include many asymptotic ends. This difference is reflected in the choice of the coordinate system. We have discussed this with Schwarzschild data in section 3. The same apply to non-extreme Kerr initial data in Weyl coordinates: $\mathcal{M}$ is singular in this coordinates. However, for extreme Kerr, the Weyl coordinates and the quasi-isotropic coordinates coincides. In this case both domains of integration coincides and $\mathcal{M}$ is finite whether $\mathcal{M}^{\prime}$ is not. 


\section{Final comments}

We have analysed the first variation of the, positive definite, mass functional $\mathcal{M}$ (defined by (46)) over axisymmetric and $(t, \phi)$ symmetric initial data with fixed angular momentum. We have shown that the critical points are the stationary, axial symmetric equations. This proves the variational principle (ii). The functional is a lower bound for the mass (inequality (47)) for all maximal, axisymmetric data. This proves (i'). In order to prove (i), and hence inequality (1), we should prove that extreme Kerr is the unique absolute minimum of $\mathcal{M}$ over axisymmetric and $(t, \phi)$ symmetric initial data with fixed angular momentum. This will require the study of the second variation of $\mathcal{M}$, given in equation (55).

\section{Acknowledgments}

It is a pleasure to thank Abhay Ashtekar, Marc Mars and Walter Simon for valuable discussions. This work has been supported by the Sonderforschungsbereich SFB/TR7 of the Deutsche Forschungsgemeinschaft.

\section{Appendix. Kerr initial data}

Consider the Kerr metric in Boyer-Lindquist coordinates $(t, \tilde{r}, \theta, \phi)$. The scalars (71) are given by

$$
\begin{aligned}
& V=\frac{\Delta-a^{2} \sin ^{2} \theta}{\Sigma}, \quad W=-\frac{2 m a \tilde{r} \sin ^{2} \theta}{\Sigma}, \\
& X=\left(\frac{\left(\tilde{r}^{2}+a^{2}\right)^{2}-\Delta a^{2} \sin ^{2} \theta}{\Sigma}\right) \sin ^{2} \theta,
\end{aligned}
$$

where

$$
\Delta=\tilde{r}^{2}+a^{2}-2 m \tilde{r}, \quad \Sigma=\tilde{r}^{2}+a^{2} \cos ^{2} \theta,
$$

and $m$ is the total mass and $a$ is the angular momentum per unit mass (i.e. $J=m a$ ).

The intrinsic metric $\tilde{h}_{a b}$ of a hypersurface $t=$ constant in these coordinates is given by

$$
\tilde{h}=\frac{\Sigma}{\Delta} \mathrm{d} \tilde{r}^{2}+\Sigma \mathrm{d} \theta^{2}+\eta \mathrm{d} \phi^{2} .
$$

The metric (A.4) has a coordinate singularity when $\Delta=0$. The solutions of the equation $\Delta=0$ are given by

$$
\tilde{r}_{+}=m+\sqrt{m^{2}-a^{2}}, \quad \tilde{r}_{-}=m-\sqrt{m^{2}-a^{2}} .
$$

By the following coordinate transformation, we extend the metric to a complete manifold with two asymptotic ends. Let us define the quasi-isotropic radius $r$ as the positive root of the following equation:

$$
\tilde{r}=r+m+\frac{m^{2}-a^{2}}{4 r} .
$$

Note that when $a=0$, this reduces to the isotropic radius for the Schwarzschild metric. The manifold (as in the Schwarzschild case) has two isometric asymptotically flat components (the region $\tilde{r} \geqslant \tilde{r}_{+}$of the metric (A.4)) joined at the minimal surface (the horizon) $\tilde{r}=\tilde{r}_{+}$. The components of $\tilde{h}_{a b}$ in the coordinates $(r, \theta, \phi)$ are given by

$$
\tilde{h}=\frac{\Sigma}{r^{2}} \mathrm{~d} r^{2}+\Sigma \mathrm{d} \theta^{2}+\eta \mathrm{d} \phi^{2} .
$$


The metric (A.7) has the form (5)-(6) with

$$
\psi^{4}=\frac{X}{\rho^{2}}, \quad \mathrm{e}^{-2 q}=\frac{\sin ^{2} \theta \Sigma}{X},
$$

where $\rho=r \sin \theta$ and $z=r \cos \theta$. Assume $m>|a|$. Then, from (A.8) we see that in the limit $r \rightarrow 0$, we have

$$
\psi=\frac{\sqrt{m^{2}-a^{2}}}{r}+\frac{m}{2 \sqrt{m^{2}-a^{2}}}+O(r), \quad \psi_{, r}=O\left(r^{-2}\right)
$$

and at infinity

$$
\psi=1+\frac{m}{2 r}+O\left(r^{-2}\right), \quad q=O\left(r^{-2}\right)
$$

From (A.8) we also have that

$$
q(\rho=0)=0 \text {. }
$$

Hence, $q$ satisfies (12), (13) and $\psi$ satisfies (14) and (24).

The velocity $\Omega$ can be calculated from equation (83) using (A.1) and (A.2):

$$
\Omega=-\frac{2 m a \tilde{r}}{\left(\tilde{r}^{2}+a^{2}\right)^{2}-\Delta a^{2} \sin ^{2} \theta} .
$$

The potential $Y$ is given by

$$
Y=2 m a\left(\cos ^{3} \theta-3 \cos \theta\right)-\frac{2 m a^{3} \cos \theta \sin ^{4} \theta}{\Sigma} .
$$

Note that equation (45) is satisfied for $z \neq 0$. To see that the integral of $\partial^{a} Y \partial_{a} Y \rho^{-4} \psi^{-8}$ over $\mathbb{R}^{3}$ is bounded, we need to check the behaviour of this function at infinity and at the axis $\rho=0$. At infinity we have

$$
\partial^{a} Y \partial_{a} Y \rho^{-4} \psi^{-8}=O\left(r^{-6}\right)
$$

and at the axis

$$
\partial^{a} Y \partial_{a} Y \rho^{-4} \psi^{-8}=O\left(r^{2}\right)
$$

where we have used (A.9). Then, the integral is bounded and therefore we have proved that the Kerr initial data satisfy our assumptions which imply that $\mathcal{M}(v, Y)=m$.

Weyl coordinates $(\bar{\rho}, \bar{z})$ are related to the coordinates $(r, \theta)$ by

$$
\bar{\rho}=\sqrt{\Delta} \sin \theta, \quad \bar{z}=(\tilde{r}-m) \cos \theta .
$$

Consider now the extreme case $m=|a|$. In this case we have

$$
r=\tilde{r}-m, \quad \Delta=r^{2},
$$

and the coordinates $(r, \theta)$ are equal to the Weyl coordinates. Equations (A.11) and (A.10) are still valid in this case. The fall-off of the conformal factor near $r=0$ is however different

$$
\psi=\frac{\sqrt{2 m}}{\left(1+\cos ^{2} \theta\right)^{1 / 4} \sqrt{r}}+O\left(r^{1 / 2}\right), \quad \psi_{, r}=O\left(r^{-3 / 2}\right),
$$

this is because $r=0$ is not an asymptotically flat in this case. Nevertheless, $\psi$ satisfies (24). The fall-of behaviour of $Y$ at infinity is the same as in the non-extreme case. Near the axis, because of (A.18), we have

$$
\partial^{a} Y \partial_{a} Y \rho^{-4} \psi^{-8}=O\left(r^{-2}\right)
$$

and hence we conclude that $\partial^{a} Y \partial_{a} Y \rho^{-4} \psi^{-8}$ is integrable over $\mathbb{R}^{3}$. 


\section{References}

[1] Ansorg M and Petroff D 2005 Black holes surrounded by uniformly rotating rings Phys. Rev. D 72024019 (Preprint gr-qc/0505060)

[2] Bardeen J M 1970 A variational principle for rotating stars in general relativity Astrophys. J. 162 71-95

[3] Bartnik R and Isenberg J 2004 The constraint equations The Einstein Equations and Large Scale Behavior of Gravitational Fields ed P T Chruściel and H Friedrich (Basel: Birkhäuser) pp 1-38 (Preprint gr-qc/0405092)

[4] Bartnik R A and Chruściel P T 2005 Boundary value problems for Dirac-type equations J. Reine Angew. Math. 579 13-73 (Preprint math.DG/0307278)

[5] Brill D 1959 On the positive definite mass of the Bondi-Weber-Wheeler time-symmetric gravitational waves Ann. Phys. 7 466-83

[6] Brill D and Lindquist R W 1963 Interaction energy in geometrostatics Phys. Rev. 131 471-476

[7] Cantor M and Brill D 1981 The Laplacian on asymptotically flat manifolds and the specification of scalar curvature Compositio Mathematica 43 317-30

[8] Carter B 1973 Black hole equilibrium states Black Holes/Les Astres Occlus (École d'Été Phys. Théor., Les Houches, 1972) (New York: Gordon and Breach) pp 57-214

[9] Dain S, Lousto C O and Takahashi R 2002 New conformally flat initial data for spinning black holes Phys. Rev. D 65104038 (Preprint gr-qc/0201062)

[10] Ernst F J 1968 New formulation of the axially symmetric gravitational field problem Phys. Rev. 167 1175-9

[11] Friedman J L and Mayer S 1982 Vacuum handles carrying angular momentum; electrovac handles carrying net charge J. Math. Phys. 23 109-15

[12] Gibbons G W and Hull C M 1982 A Bogomolny bound for general relativity and solitons in $N=2$ supergravity Phys. Lett. B 109 190-4

[13] Gibbons G W, Hull C M and Warner N P 1983 The stability of gauged supergravity Nuclear Physics B 218 173-90

[14] Hawking S W 1973 A variational principle for black holes Commun. Math. Phys. 33 $323-34$

[15] Horowitz G T 1984 The positive energy theorem and its extensions Asymptotic Behavior of Mass and Spacetime Geometry (Corvallis, Oregon, 1983) (Lecture Notes in Physics vol 202) ed F J Flaherty (Berlin: Springer) pp $1-21$

[16] Horowitz G T 2005 Higher dimensional generalizations of the Kerr black hole Preprint gr-qc/0507080

[17] Matzner R A and Misner C W 1967 Gravitational field equations for sources with axial symmetry and angular momentum Phys. Rev. 154 1229-32

[18] Maxwell D 2005 Solutions of the Einstein constraint equations with apparent horizon boundaries Commun. Math. Phys. 253 561-83 (Preprint gr-qc/0307117)

[19] Neugebauer G and Herold H 1992 Gravitational fields of rapidly rotating neutron stars: theoretical foundation Relatividtic Gravity Research (Lectures Notes in Physics vol 410) ed J Ehlers and G Schäfer (Berlin: Springer) pp 303-18

[20] Szabados L B 2004 Quasi-local energy-momentum and angular momentum in GR: A review article Living Rev. Relativ. 7 http://www.livingreviews.org/lrr-2004-4 (cited on 8 August 2005)

[21] Wald R M 1984 General Relativity (Chicago, IL: The University of Chicago Press) 